\title{
Estruturas de Interação na Psicoterapia Psicodinâmica de uma Menina com Transtorno de Adaptação
}

\author{
Fernanda Munhoz Driemeier Schmidt ${ }^{1, *}$ \\ Orcid.org/0000-0003-3072-9671 \\ Marina Bento Gastaud ${ }^{1}$ \\ Orcid.org/0000-0002-1760-7154 \\ Vera Regina Rohnelt Ramires ${ }^{1}$ \\ Orcid.org/0000-0002-1760-7154
}

${ }^{1}$ Universidade do Vale do Rio dos Sinos, São Leopoldo, RS, Brasil

\section{Resumo}

A literatura científica aponta a necessidade de estudos com foco no processo e nos resultados da psicoterapia psicodinâmica de crianças. Além disso, o relacionamento e as estruturas de interação entre terapeuta e paciente têm sido enfatizadas como importante fator de mudança terapêutica. O objetivo desse estudo foi analisar as estruturas de interação na psicoterapia psicodinâmica de uma criança com transtorno de adaptação. Realizou-se um estudo descritivo, longitudinal, baseado no procedimento de estudo de caso sistemático. Os participantes foram uma menina de oito anos e sua terapeuta. As 40 sessões de psicoterapia foram analisadas através do Child Psychotherapy Q-Set (CPQ). Foram identificadas cinco estruturas de interação, com base na análise fatorial de componentes principais com rotação Varimax. Elas descrevem diferentes padrões de interação, com utilização de abordagens suportivas, diretivas e interpretativas. A importância das estruturas de interação como vetor de mudança, variáveis do terapeuta e uma tendência de abordagens integrativas na psicoterapia de crianças foram discutidas.

Palavras-chave: Psicoterapia de crianças, estudo de caso único, pesquisa de processo, estruturas de interação.

\section{Interaction Structures in the Psychodynamic Psychotherapy of a Girl Diagnosed with Adjustment Disorder}

\begin{abstract}
The scientific literature indicates the need for studies focusing on the process and outcomes of child psychodynamic psychotherapy. In addition, the relationship and the interaction structures between therapist and patient have been emphasized as an important factor of therapeutic change. The aim of this study was to analyze interaction structures in the psychodynamic psychotherapy of a child with ad-
\end{abstract}

Endereço para correspondência: Casemiro de abreu, 370, apto 402, Bairro Rio Branco, Porto Alegre, RS, Brasil. E-mail: fernandadriemeier@hotmail.com

Apoio financeiro: CAPES - Coordenação de Aperfeiçoamento de Pessoal de Nível Superior; CNPq - Conselho

Nacional de Desenvolvimento Científico e Tecnológico. 
justment disorder. We conducted a descriptive, longitudinal study based on the systematic case study procedure. The participants were an 8-year-old girl and her therapist. Forty psychotherapy sessions were analyzed using the Child Psychotherapy Q-Set (CPQ). Five interaction structures were identified on the basis of factor analysis of main components with varimax rotation. They describe different patterns of interaction, using supportive, directive, and interpretive approaches. The importance of interaction structures as a vector of change, therapist variables, and the trend toward integrative approaches in child psychotherapy are discussed.

Keywords: Child psychotherapy, single case study, process research, interaction structures..

\section{Estructuras de Interacción en la Psicoterapia Psicodinámica de una Niña con Trastorno Adaptativo}

\section{Resumen}

La literatura científica indica la necesidad de estudios con foco en el proceso y en los resultados de psicoterapia psicodinámica de niños. Además, la relación y las estructuras de interacción entre terapeuta y paciente se han enfatizado como un importante factor de cambio terapéutico. El objetivo de este estudio fue analizar las estructuras de interacción en la psicoterapia psicodinámica de una niña con trastorno de adaptación. Se realizó un estudio descriptivo, longitudinal basado en lo método de estudio sistemático de casos. Los participantes fueron una niña de ocho años y su terapeuta. Las Cuarenta sesiones de psicoterapia fueran analizadas mediante el Child Psychotherapy Q-Set (CPQ). Fueran identificadas cinco estructuras de interacción, con base en el análisis factorial de componentes principales con rotación Varimax. Estas describen diferentes patrones de interacción, con utilización de enfoques suportivos, directivos e interpretativos. La importancia de las estructuras de interacción como un vector de cambio, las variables del terapeuta y la tendencia de los enfoques de integración en psicoterapia de niños fueron discutidos.

Palabras clave: Psicoterapia de niños, estudio de caso único, proceso de búsqueda, estructuras de interacción.

O Transtorno de Adaptação (TA) em crianças é descrito como uma dificuldade de adaptação a uma nova situação da vida da criança, em resposta a um evento estressor identificável que resulta no desenvolvimento de sintomas emocionais, somáticos e/ou comportamentais. Os estressores podem ser recorrentes ou contínuos e podem afetar apenas o indivíduo ou a família inteira. Com frequência, os estressores são eventos da vida cotidiana da criança e os sintomas gerados indicam que ela está em um estado de sofrimento maior do que aquele que seria esperado, pela exposição ao estressor, causando um comprometimento significativo no funcionamento social ou escolar. A sintomatologia deve ocorrer dentro de um mês após o evento estressante (American Psychiatric Association [APA], 2013).
Três fatores são fundamentais para a compreensão dos TA: a natureza do estressor, o sentido consciente e inconsciente deste e a vulnerabilidade pré-existente do paciente. Uma maior suscetibilidade está associada à perda de um dos pais na infância ou à criação em uma família disfuncional. $\mathrm{O}$ apoio real ou percebido por pessoas próximas e importantes pode afetar as respostas comportamentais e emocionais aos estressores (Sadock \& Sadock, 2007).

A psicoterapia parece ser a primeira escolha para os TA (Sadock \& Sadock, 2007), porém os estudos que fundamentam tal conduta clínica são escassos e pouco precisos. A psicoterapia individual deve oferecer oportunidades de explorar o sentido do estressor para elaboração de traumas. É necessário, também, que a psicoterapia ajude o paciente a se adaptar aos estressores que não 
são reversíveis nem têm duração limitada. Nesse sentido, a psicoterapia psicodinâmica (PP) pode ser indicada (Kramer, De Roten, Michel, \& Despland, 2009), já que é um processo que visa instrumentalizar o indivíduo a um aumento do conhecimento sobre seu funcionamento, o que resulta no uso de defesas mais maduras para lidar com conflitos psíquicos, na melhora do padrão das relações objetais (Eizirik \& Hauck, 2007) e em mudanças sintomáticas significativas (Williams, 2007).

Visto que as psicoterapias desempenham um papel importante na melhora do TA, destaca-se a importância do estudo dos tratamentos destas psicopatologias, principalmente no que tange aos ingredientes ativos de mudança terapêutica (Midgley, 2007). Partindo dessas premissas, este estudo descreve uma investigação empírica do processo terapêutico psicodinâmico de uma menina de oito anos, com diagnóstico de TA, e mais especificamente as estruturas de interação que se estabeleceram entre paciente e terapeuta. $\mathrm{O}$ estudo desse tema vai ao encontro da necessidade de pesquisas empíricas que demonstrem de que forma ocorre a efetividade do tratamento psicodinâmico com crianças.

\section{O Processo Psicoterapêutico Psicodinâmico de Crianças e as Estruturas de Interação}

Embora a pesquisa de processo de psicoterapia de crianças tenha se expandido nos últimos anos, o número de estudos que exploram os mecanismos de mudança é muito baixo. Os estudos disponíveis têm contemplado a discussão de medidas de resultado como comportamento da criança, o jogo, as defesas, suas relações objetais, a relação terapêutica, porém sem capturar integralmente o processo do encontro terapêutico em sua complexidade (Midgley, 2007; Midgley \& Kennedy, 2011).

Diante dessa necessidade de conhecer-se mais a natureza da ação terapêutica e do processo terapêutico, Jones (2000) desenvolveu, com base na Metodologia $Q$ (Couto, Farate, Ramos, \& Fleming, 2011), o Psychotherapy Process $Q$ -Set (PQS). O PQS é considerado um instrumento útil por possibilitar uma descrição qualitativa e quantitativa do processo terapêutico, tendo sido desenvolvido para ser usado em estudos empíricos com adultos (Jones, 1988, 2000).

Jones (2000) utilizou o PQS para analisar as estruturas de interação (EI) na relação terapêutica, que são padrões repetitivos de interação que ocorrem entre paciente e terapeuta, às vezes sem o conhecimento consciente dos mesmos. Tal concepção segue uma linha de pensamento que enfatiza a interação como força propulsora da mudança terapêutica, em que fatores interpessoais e de relacionamento da dupla poderão favorecer a retomada do desenvolvimento emocional do paciente. Jones (2000) argumenta que só a investigação desses processos de interação específicos pode identificar os ingredientes que produzem mudança terapêutica. $\mathrm{O}$ conhecimento dessas estruturas pode auxiliar os psicoterapeutas na prática clínica, permitindo saber o que pode ser esperado de pacientes com determinados sintomas e comportamentos e de como as interações podem mudar ao longo do tempo (Goodman \& Athey-Lloyd, 2011).

O estudo das EI auxilia no entendimento de aspectos intersubjetivos, de transferência e contratransferência, actings out entre outros fenômenos do processo terapêutico, revelando os conflitos do paciente e as reações e intervenções do terapeuta frente a esses conflitos. Assim, a vivência da relação terapêutica e a compreensão do significado das EI, constituem componentes importantes para a compreensão dos ingredientes ativos e dos mecanismos de mudança nas psicoterapias psicodinâmicas (Jones, 2000). As transformações ocorridas, ao longo do tempo, nessas interações, foram relacionadas às mudanças na estrutura psicológica dos pacientes e à melhora sintomática dos mesmos (Ablon \& Jones, 2005; Jones, 2000).

Seguindo os passos de Jones (2000), diversos autores se debruçaram sobre o tema do estudo do processo terapêutico e suas EI com pacientes adultos, abrangendo diferentes patologias (Ablon \& Jones, 2005; Coombs, Coleman, \& Jones, 2002; Goodman, Edwards, \& Chung, 2014; Jones, 2000; Jones \& Pulos, 1993). Nessa direção, juntamente com a necessidade crescente de aprofundar os estudos na psicoterapia de 
crianças, Schneider e Jones (2004) desenvolveram, a partir do PQS, o Child Psychotherapy $Q$-set (CPQ), um instrumento equivalente que permite a descrição do processo terapêutico com crianças (Schneider, 2004; Schneider \& Jones, 2004, 2012). Assim, algumas pesquisas passaram a ser realizadas no campo da psicoterapia de crianças utilizando esse instrumento (Goodman \& Athey-Lloyd, 2011; Ramires, Carvalho, Schmidt, Fiorini, \& Goodman, 2015; Schneider, 2004; Schneider, Midgley, \& Duncan, 2010; Schneider, Pruetzel-Thomas, \& Midgley, 2009).

O CPQ vem possibilitando, portanto, o estudo do processo terapêutico com crianças e a análise das EI. Schneider et al. (2009) analisaram quatro psicoterapias de orientação psicodinâmica e duas com abordagem cognitivo-comportamental. Os pacientes tinham entre 8 e 12 anos de idade e estavam em tratamento por sintomas de ansiedade e/ou depressão. Os resultados obtidos com base no CPQ mostraram que existem semelhanças nas características do processo psicoterapêutico de crianças com o mesmo tipo de dificuldade, mesmo quando atendidas por diferentes terapeutas. Contrariamente, o mesmo terapeuta com diferentes crianças que apresentavam dificuldades distintas apresentou padrões de interação diferentes. Em ambos os tratamentos, psicodinâmico e cognitivo comportamental, os itens relativos à criança do CPQ foram bastante similares, o que demonstra que as crianças se apresentaram de forma homogênea, talvez porque sua idade, tipo de distúrbio e grau de dificuldade eram similares. Quanto aos itens do CPQ relativos ao terapeuta, as técnicas que caracterizam a PP foram notavelmente ausentes na psicoterapia cognitivo comportamental, e vice-versa.

Posteriormente, Schneider et al. (2010) descreveram o processo terapêutico de uma menina de 11 anos de idade, que buscou psicoterapia por apresentar diagnóstico de Transtorno Depressivo Maior, Transtornos de Ansiedade Generalizada, Transtorno do Pânico e Transtorno Evitativo da Infância pelo DSM-IV. O tratamento durou três anos, com frequência de quatro sessões semanais e foram identificadas as EI e descritos os resultados obtidos. Foram utilizadas para a análise do processo terapêutico nove sessões (três do início, três do meio e três do final do tratamento). Foram identificadas três EI, 1- Trazendo para fora a criança isolada, 2- Trabalhando com estados de ansiedade e resistência, 3-Saindo da Concha. Segundo os autores, a soma de intervenções interpretativas e suportivas favoreceu a melhora sintomática da paciente.

Goodman e Athey-Lloyd (2011) analisaram se as EI diferem quando o paciente é constante e o terapeuta muda no decorrer de um tratamento. Para isso, analisaram dois anos de PP de uma criança de seis anos de idade com Transtorno de Asperger. Em cada ano, a criança foi atendida por um terapeuta diferente e a análise do processo foi baseada no CPQ. Foram identificadas quatro EIs: 1 -Terapeuta não diretivo, suportivo e tranquilizador com uma criança complacente, curiosa, construindo insight e sentimentos positivos; 2 -Terapeuta confiante, mentalizador e cooperativo com uma criança expressiva, confortável e em busca de ajuda; 3 - Terapeuta crítico e não sintonizado com uma criança distante, emocionalmente desconectada e incompreendida; e 4-Terapeuta acolhedor com criança brincalhona e competitiva. As EI encontradas tiveram magnitudes diferentes entre os terapeutas e também ao longo do tempo de cada ano de tratamento. Os resultados confirmaram as hipóteses dos autores de que as EI, além de flutuarem através do tempo, também difeririam entre dois terapeutas atendendo a mesma criança.

Goodman (2015) examinou as EI na PP de uma criança diagnosticada com transtorno de personalidade borderline e tratada por dois terapeutas. Quatro EI puderam ser identificadas: 1 - Terapeuta sensivel e acolhedora com criança motivada, com capacidade de insight e atitude positiva em relação à terapeuta, 2 - Terapeuta interpretativa com criança passiva-agressiva, 3 - Terapeuta confiante e bem-humorada com criança animada e brincalhona, 4 - Terapeuta estruturante e flexivel com criança dificil e irritada. As EIs diferiram na sua magnitude entre os dois terapeutas e também diferiram ao longo do tempo em cada tratamento. $\mathrm{O}$ autor discute que o que pode ser eficaz em uma díade pode não funcionar em outra devido a características específicas do terapeuta e da díade. 
Ramires et al. (2015) também analisaram as EI na PP de um menino com Transtorno de Asperger, além de possíveis mudanças em sua organização psíquica. Aproximadamente $30 \mathrm{me}-$ ses de psicoterapia foram analisados com CPQ. Foram identificadas quatro EI: 1- Criança ativa, confiante, animada, competindo com uma terapeuta conectada, com postura mentalizadora e receptiva, 2 - Criança retraída e defensiva, com terapeuta insegura, não responsiva e didática, 3 - Terapeuta receptiva com criança exigente, provocativa e hostil, 4 - Terapeuta tranquilizadora, suportiva e não diretiva com criança complacente e não espontânea. Duas EI variaram ao longo do tempo. Uma EI se mostrou semelhante ao estudo de Goodman e Athey-Lloyd (2011).

Constata-se que o estudo das EI na PP de crianças vem trazendo contribuições para a compreensão do processo desses tratamentos, com crianças que apresentam diferentes problemas clínicos. A necessidade de estudos no campo da psicoterapia de crianças, que contemplem seus resultados e processo também tem sido largamente reconhecida. Sendo assim, o objetivo deste estudo foi descrever e analisar o processo terapêutico de uma menina com diagnóstico de TA, identificando e compreendendo as EI estabelecidas no decorrer desse tratamento.

\section{Método}

\section{Participantes}

Alice (pseudônimo), tinha oito anos de idade no início de sua psicoterapia e cursava o terceiro ano do Ensino Fundamental. Foi diagnosticada com TA com ansiedade (309.24), de acordo com o DSM-5 (APA, 2013), pela sua terapeuta e pelo supervisor do caso. Ela residia com sua mãe e irmã, pois seu pai havia falecido seis meses antes da busca por atendimento, de forma repentina e inesperada. Consequentemente, ocorreram mudanças na estrutura familiar, causando a troca de escola das filhas e a necessidade da mãe dedicar-se mais ao trabalho. Alice era bastante apegada ao pai, e ele a ela. A mãe procurou atendimento para a filha, encaminhada pela neuropediatra, por ela apresentar crises de enxaqueca, de choro e dores de estômago, sem causas orgânicas. Ali- ce dizia não gostar de ir à escola e não conseguia fazer amigos. Segundo a mãe e a professora, Alice era uma criança carinhosa, perfeccionista e que não tolerava errar, ficando ansiosa quando chamavam sua atenção. A terapeuta concordava com essa descrição, percebendo em diversos momentos, ao longo da terapia, a vontade de menina em agradar e ser elogiada e querida. Em suas sessões, Alice demonstrava capacidade de reflexão e criatividade nas brincadeiras, usando o jogo simbólico. A criança e sua mãe concordaram em participar da pesquisa, consentindo a filmagem das sessões da menina.

A psicoterapeuta possuía 10 anos de experiência clínica quando foi procurada pela paciente. Cursou especialização em PP de crianças e doutorado na mesma área. Realizava supervisão clínica do caso em estudo e estava em tratamento analítico. Concordou em participar do estudo previamente.

Durante o processo terapêutico, a terapeuta engravidou e teve bebê. Quando retornou da sua licença maternidade, após dois meses e meio de interrupção, a mãe de Alice solicitou alta do tratamento, por considerar que a filha estava melhor, e por dificuldades de manter o tratamento. Um familiar próximo, que fazia parte da sua rede de apoio, havia adoecido gravemente, e ela se sentia sobrecarregada com todos os compromissos e demandas que tinha. Alice, de fato, havia superado os sintomas que a trouxeram para psicoterapia, mas havia passado a demonstrar alguns traços obsessivos que, na visão da terapeuta, ainda requeriam atenção. A menina desejava manter o tratamento, mas expressou que se sentia melhor. Disse: "agora eu consigo botar pra fora o que eu sinto. Quando eu cheguei aqui, eu era como um passarinho preso numa gaiola. Agora é como se tivesse saído pra fora". Sendo assim, diante das dificuldades apresentadas pela mãe, foi combinado um período de aproximadamente dois meses, com sessões quinzenais, para que fosse trabalhado o encerramento do processo.

\section{Delineamento}

Foi realizado um estudo naturalístico, descritivo, longitudinal, baseado no método de Estudo de Caso Sistemático (ECS; Edwards, 
2007). O ECS propõe-se a estudar em profundidade um caso, sendo baseado em procedimentos desenvolvidos no contexto clínico ou naturalista, objetivando a compreensão dos fatores que contribuem para a mudança no processo psicoterápico e os resultados das intervenções clínicas.

\section{Instrumentos}

Child Psychotherapy Q - Set (CPQ; Schneider \& Jones, 2012). O CPQ foi construído baseado no PQS (Jones, 2000) e foi traduzido e adaptado para o português brasileiro (Ramires \& Schneider, 2016). Trata-se de um procedimento que visa a análise do processo psicoterapêutico com crianças de 3 a 13 anos de idade. É composto por 100 itens com afirmações que descrevem três domínios do processo terapêutico: 1- Itens que descrevem as atitudes, sentimentos, comportamento ou experiência da criança, 2 - Itens que refletem as ações e atitudes do psicoterapeuta $\mathrm{e} 3$ - Itens referentes à natureza da interação da díade. Durante o curso do tratamento, esses itens tendem a agrupar-se e em conjunto descreverem estruturas repetitivas de interação (Jones, 2000).

Após assistir ao vídeo da sessão terapêutica, juízes treinados ordenam, de maneira independente, através de uma distribuição forçada (ipsativa), os 100 itens em 9 categorias para descrever a sessão em um continuum que varia entre o menos característico ao mais característico de cada sessão terapêutica. Essa distribuição ipsativa proporciona que os juízes comparem os itens entre si e os coloquem em uma distribuição normal, com a maioria dos itens colocados no centro e menos itens nos extremos. Métodos ipsativos possuem benefícios para avaliação psicológica, pois o formato de escolha forçada reduz a influência da desejabilidade social e o viés de resposta uniforme nos resultados das avaliações (Cheung \& Chan, 2002; Christiansen, Burns, \& Montgomery, 2005; Miller \& Lovler, 2015).

A fidedignidade e validade do CPQ vêm sendo demonstradas por diferentes investigações. Sua validade discriminante foi demonstrada em alguns estudos (Goodman, Midgley, \& Schneider, 2015; Schneider et al., 2009), assim como a fidedignidade interavaliadores (Goodman \& Athey-Lloyd, 2011; Goodman et al., 2015; Ra- mires et al., 2015; Schneider, 2004; Schneider et al., 2010). Em todos eles, análises independentes de grupos de sessões de psicoterapia de crianças realizadas por avaliadores treinados têm atingido índices de concordância superiores a 0,70 (correlação intraclasse).

\section{Procedimentos}

Na busca de tratamento pela mãe da criança, foi realizada uma avaliação clínica, para confirmar o diagnóstico e a indicação de psicoterapia. A psicoterapia teve enfoque psicodinâmico, na perspectiva da teoria das relações objetais, foi realizada em consultório particular, com frequência de uma sessão semanal, com duração de 50 minutos. No total, foram realizadas, filmadas e analisadas 40 sessões de psicoterapia.

Cada sessão do tratamento de Alice foi codificada por dois juízes, de forma independente, formando pares aleatórios e que se intercambiaram. A equipe de juízes foi composta por seis psicólogos, treinados para o uso do CPQ, e com experiência clínica em PP. A concordância entre qualquer par de juízes foi de pelo menos 0,70 (alpha de Cronbach) e variou de 0,69 a 0,90 ( $m=$ $0,80$ e $D P=0,05)$ nas 40 sessões de Alice. Com as classificações feitas pelos pares de juízes, calculou-se a média de cada sessão para formar escores compostos utilizados em todas as análises subsequentes.

Primeiramente, a média dos escores compostos das 40 sessões permitiu identificar o tom geral do tratamento de Alice, evidenciando os itens mais e menos característicos, de acordo com o CPQ. Numa segunda etapa, os escores compostos das 40 sessões foram submetidos a uma análise fatorial de componentes principais com rotação varimax, para identificação das EI. Correlações de Pearson foram utilizadas para analisar mudanças nas EI resultantes ao longo do tempo. Essas análises utilizaram o software SPSS 23.0.

\section{Resultados}

O exame da média dos itens mais e menos característicos do CPQ em todas as 40 sessões de psicoterapia de Alice descrevem característi- 


\section{Tabela 1}

Dez Itens do CPQ Mais e Menos Característicos do Processo de Psicoterapia de Alice

\begin{tabular}{|c|c|c|}
\hline Itens do CPQ & Média & $D P$ \\
\hline \multicolumn{3}{|l|}{ Itens mais característicos } \\
\hline 31 - T solicita mais informação ou elaboração. & 8,28 & 0,57 \\
\hline 6 - T é sensível aos sentimentos da C. & 8,20 & 0,55 \\
\hline 28 - T percebe acuradamente o processo terapêutico. & 8,06 & 0,65 \\
\hline 3 - Os comentários do $\mathrm{T}$ visam a encorajar a fala da $\mathrm{C}$. & 7,70 & 0,79 \\
\hline 88 - O material da sessão é significativo e relevante em relação aos conflitos da C. & 7,56 & 0,65 \\
\hline 76 - T faz ligações entre sentimentos e experiências da C. & 7,45 & 0,78 \\
\hline 77 - A interação do T com a C é sensível ao seu nível de desenvolvimento. & 7,35 & 0,85 \\
\hline 23 - A sessão terapêutica tem um tema ou foco específico. & 7,25 & 0,68 \\
\hline 86 - T é confiante, seguro de si [vs. incerto ou inseguro]. & 7,25 & 0,76 \\
\hline 82 - $\mathrm{T}$ ajuda $\mathrm{C}$ a manejar os sentimentos. & 7,16 & 0,98 \\
\hline \multicolumn{3}{|l|}{ Itens menos característicos } \\
\hline 9 - T não é responsivo [vs. afetivamente engajado]. & 1,12 & 0,25 \\
\hline 41 - C não se sente compreendida pelo $\mathrm{T}$. & 1,62 & 0,69 \\
\hline 40 - C se comunica sem afeto. & 1,72 & 0,48 \\
\hline 5 - C tem dificuldade para compreender os comentários do $\mathrm{T}$. & 1,73 & 0,54 \\
\hline 18 - T é crítico e transmite falta de aceitação. & 1,77 & 0,59 \\
\hline 56 - C está distante dos seus sentimentos. & 1,91 & 0,45 \\
\hline 17 - T ativamente exerce controle sobre a interação (ex. estruturando, introduzindo novos tópicos). & 1,97 & 0,86 \\
\hline 26 - C é socialmente não sintonizada ou inapropriada. & 2,02 & 0,47 \\
\hline 42 - C ignora ou rejeita os comentários e observações do T. & 2,15 & 0,46 \\
\hline $\begin{array}{l}58 \text { - C parece relutante em examinar pensamentos, reações ou motivações relacionadas } \\
\text { aos problemas. }\end{array}$ & 2,43 & 0,99 \\
\hline
\end{tabular}

Nota $. \mathrm{C}=$ criança, $\mathrm{T}=$ terapeuta.

cas gerais desse processo terapêutico. Percebe-se que os itens mais característicos se referiram ao comportamento e atitudes da terapeuta e, em contrapartida, os itens menos característicos referiram-se ao comportamento e atitudes da criança (Tabela 1).

Um olhar sobre os itens com maiores e menores médias sugere que este tratamento possui características psicodinâmicas e foi conduzido por uma terapeuta sensível, afetivamente engajada, confiante e sintonizada com os estados emocionais da criança. A terapeuta não dirigia nem estruturava a sessão, procurava encorajar a expressão dos sentimentos da paciente e ajudá-la a manejar seus sentimentos. As comunicações de Alice eram carregadas de afeto e ela se sentia compreendida pela terapeuta, aceitando suas intervenções, mostrando-se disposta a examinar seus pensamentos e sentimentos.

A análise fatorial de componentes principais permitiu identificar cinco EI conceitualmente interpretáveis, que representaram $36,93 \%$ da variância do tratamento, conforme Tabela 2 e 3. Este resultado é consistente com outros estudos (Goodman \& Athey-Lloyd, 2011; Goodman et al., 2015; Ramires et al., 2015). 
Tabela 2

Estruturas de Interação 1, 2 e 3

Estrutura de Interação 1 - Terapeuta sintonizada e com postura interpretativa com criança ativa, expressiva e demandante. $(\alpha=0,81)$

Itens do CPQ

Carga do Fator

28 - T percebe acuradamente o processo terapêutico.

0,68

72 - C é ativa.

0,66

29 - A qualidade do brincar da C é fluida, absorta [vs. fragmentada, esporádica].

0,60

96 - Os pais da C são um tópico de discussão.

$-0,59$

69 - A situação de vida atual ou recente da C é enfatizada.

$-0,54$

46 - T interpreta o significado do jogo da C.

0,54

22 - C expressa medos de ser punida ou ameaçada.

$-0,52$

75 - Interrupções, pausas no tratamento ou o término da terapia são discutidos.

0,50

13 - C está animada ou excitada.

0,50

83 - $\mathrm{C}$ é exigente.

0,46

90 - Os sonhos ou fantasias da C são discutidos.

$-0,45$

39 - C é competitiva, rivaliza com $\mathrm{T}$.

0,45

73 - C expressa medos ou mostra um comportamento fóbico.

$-0,43$

Estrutura de Interação 2 - Terapeuta sensível e suportiva com criança retraída e distante. $(\alpha=0,85)$

Itens do CPQ

Carga do Fator

99 - T oferece ajuda ou orientação.

12 - T modela emoções não ditas ou não elaboradas.

$-0,72$

77 - A interação do T com a C é sensível ao seu nível de desenvolvimento.

0,62

27 - Há um foco em ajudar C a planejar seu comportamento fora da sessão.

0,60

53 - C transmite consciência das próprias dificuldades internas.

$-0,56$

71 - C se engaja no jogo de faz-de-conta.

$-0,56$

91 - Uma fase anterior do desenvolvimento é um tópico.

$-0,54$

26 - C é socialmente não sintonizada ou inapropriada.

$-0,53$

81 - T enfatiza sentimentos para ajudar C a experimentá-los mais profundamente.

$-0,52$

54 - C é clara e organizada na sua expressão verbal.

0,51

95 - O brincar da C carece de espontaneidade.

0,51

44 - C se sente cautelosa ou desconfiada (vs. confiante e segura).

0,50

10 - C procura maior intimidade com $\mathrm{T}$.

$-0,48$

40 - C se comunica sem afeto.

0,47

93 - T é neutro.

$-0,45$

47 - Quando a interação com C é difícil, T tenta se ajustar à C.

0,42

86 - T é confiante, seguro de si [vs. incerto ou inseguro].

0,42 
Estrutura de Interação 3 - Terapeuta didática e diretiva com criança agressiva e defensiva (projetiva). $(\alpha=0,83$ ) Itens do CPQ Carga do Fator

34 - C culpa os outros, ou forças externas, pelas dificuldades. 0,69

55 - T recompensa diretamente comportamentos desejáveis.

66 - T é diretamente tranquilizador.

85 - A agressividade da $\mathrm{C}$ é direcionada para ela mesma.

65 - T clarifica, reafirma, ou reformula comunicações da C.

70 - C se esforça para controlar sentimentos ou impulsos.

87 - $\mathrm{T}$ informa a $\mathrm{C}$ do impacto potencial do seu comportamento sobre os outros (não incluindo T).

84 - C expressa raiva ou sentimentos agressivos.

38 - T e C demonstram um vocabulário ou compreensão compartilhada quando se referem

82 - T ajuda $\mathrm{C}$ a manejar os sentimentos.

57 - T tenta modificar distorções nas crenças da C.

51 - C atribui as próprias características ou sentimentos ao T.

Nota. $\mathrm{C}=$ criança, $\mathrm{T}=$ terapeuta.

\section{Tabela 3}

Estruturas de Interação 4 e 5

Estrutura de Interação 4 - Terapeuta diretivo com criança dependente, resistente e envergonhada. $(\alpha=0,78)$

Itens do CPQ

Carga do Fator

100 - T faz conexões entre a relação terapêutica e outros relacionamentos.

67 - T interpreta os desejos, sentimentos ou ideias rejeitados ou inconscientes.

32 - C alcança uma nova compreensão ou insight.

49 - C transmite ou expressa sentimentos confusos ou conflituosos sobre T.

80 - C se comporta de uma maneira dependente [vs. insiste na independência].

58 - C parece relutante em examinar pensamentos, reações ou motivações relacionadas aos problemas.

92 - Os sentimentos ou percepções da C são relacionados a situações ou comportamentos do passado.

9 - T não é responsivo [vs. afetivamente engajado].

42 - C ignora ou rejeita os comentários e observações do T.

17 - T ativamente exerce controle sobre a interação (ex. estruturando, introduzindo novos tópicos). 
59 - C se sente inadequada e inferior [vs. eficaz e superior].

0,41

45 - T tolera os fortes afetos ou impulsos da C.

$-0,40$

Estrutura de Interação 5 - Terapeuta receptivo e apoiador com criança ansiosa. $(\alpha=0,76)$

Itens do CPQ

Carga do Fator

31 - T solicita mais informação ou elaboração.

2 - T comenta sobre o comportamento não verbal da C (ex. postura corporal, gestos).

$-0,54$

4 - Há discussão sobre por que a C está em terapia.

0,54

36 - T assinala o uso de defesas por parte da $\mathrm{C}$.

$-0,51$

79 - T comenta as mudanças no humor ou nas emoções da C.

$-0,51$

64 - $\mathrm{C}$ inclui $\mathrm{T}$ no jogo.

$-0,50$

63 - C explora relacionamentos com outros significativos.

0,50

50 - T chama a atenção para sentimentos considerados inaceitáveis pela $\mathrm{C}$ (ex. raiva, inveja, ou excitação).

52 - T faz afirmações explícitas sobre o final da sessão, o próximo final de semana ou as férias.

43 - T sugere o significado do comportamento dos outros.

0,42

7 - C está ansiosa e tensa [vs. calma e descontraída].

0,42

16 - Há discussão ou evidência de funções corporais (ex. movimentos intestinais).

$-0,40$

Nota $. \mathrm{C}=$ criança, $\mathrm{T}=$ terapeuta.

EI 1 - Terapeuta sintonizada e com postura interpretativa com criança ativa, expressiva $e$ demandante $(\alpha=0,81)$ : nessa estrutura de interação, a terapeuta faz uma análise acurada do processo terapêutico e a criança está calma e à vontade, brincando de forma fluída durante a sessão, competindo com a terapeuta e sentindo-se aceita e acolhida. Separações e interrupções no processo de tratamento foram discutidas. Essa estrutura tornou-se mais característica ao longo das 40 sessões do tratamento $(r=0,365, p$ $<0,05$; Figura 1).

EI 2 - Terapeuta sensivel e suportiva com criança retraída e distante $(\alpha=0,85)$ : essa EI reflete uma interação sensível ao nível de desenvolvimento e às necessidades da menina. Alice apresentava cautela na relação com a terapeuta, com um jogo concreto e limitado pelas regras. A terapeuta mostrava-se confiante, evidenciando uma postura de apoio e suportiva. A criança expressava-se de forma clara e fluente, porém não parecia ter consciência de suas dificuldades internas. A EI 2 não apresentou alterações significativas ao longo das 40 sessões $(r=0,061)$.
EI 3 - Terapeuta didática e diretiva com criança agressiva e defensiva (projetiva; $\alpha=$ 0,83): Alice mostrava-se resistente nessa EI, expressando e direcionando a raiva e/ou sentimentos agressivos para fora, não parecendo fazer esforço para conter ou regular os sentimentos que ela estava experimentando e nem mostrando curiosidade sobre os mesmos. Ela atribuía suas características ou sentimentos à terapeuta, que buscava enfatizar os estados internos e afetos da paciente, com o objetivo de tornar os seus significados mais evidentes, encorajando a criança a explorar e verbalizar pensamentos e sentimentos. Nessa EI, a menina culpava os outros pelas dificuldades, enquanto a terapeuta era diretamente tranquilizadora, tentando modificar distorções nas suas crenças. Essa EI não apresentou alterações significativas ao longo das 40 sessões $(r=0,190)$.

EI 4 - Terapeuta diretivo com criança dependente, resistente e envergonhada $(\alpha=$ $0,78)$ : nessa EI a paciente se mostrava resistente em examinar o seu próprio papel nos problemas, expressando sentimentos confusos 
sobre a terapeuta, apesar de adotar um comportamento dependente. Expressava também sentimentos de inadequação e inferioridade, sentindo-se tímida e envergonhada. Houve rejeição das observações ou interpretações da terapeuta por parte de Alice nessa EI, e mais colocação de limites e menor responsividade por parte da terapeuta. As experiências atuais e passadas foram discutidas, mas não relacionadas. A atividade da terapeuta, durante esses períodos, não incluía tentativas de relacionar os aspectos interpessoais da terapia com experiências de outros relacionamentos, mesmo quando existiam oportunidades para fazer isso. Essa estrutura tornou-se significativamente menos característica ao longo das 40 sessões de psicoterapia de Alice $(r=-0,545, p<0,01$; Figura 2).

EI 5 - Terapeuta receptivo e apoiador com criança ansiosa. $(\alpha=0,76)$ : nessa EI a terapeuta buscava informações ou estimulava a criança para uma descrição mais detalhada dos seus sentimentos e experiências, tentando interpretar o significado do comportamento das pessoas na vida dela. Alice mostrava-se ansiosa e tensa, porém explorando suas experiências de relacionamento com outros significativos. A terapeuta não focalizava o comportamento não verbal $\mathrm{e}$ repetitivo, ou sentimentos e reações difíceis de lidar para Alice. As razões para a terapia eram discutidas, assim como as separações. Essa EI não apresentou alterações significativas ao longo das 40 sessões de psicoterapia $(r=0,208)$.

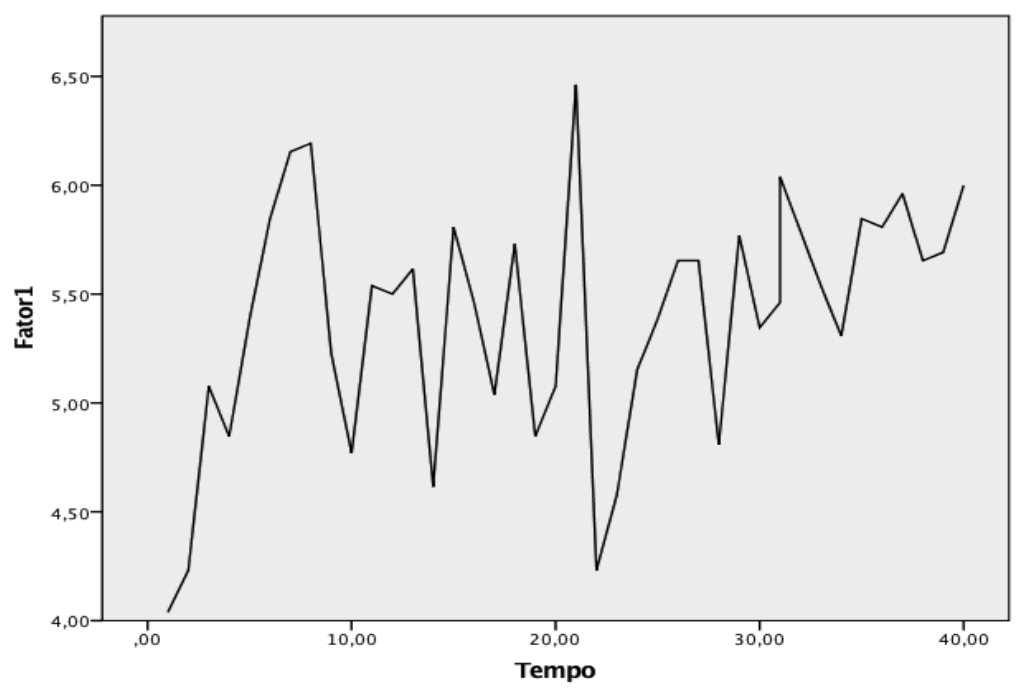

Figura 1. Variação da estrutura de interação 1 ao longo das 40 sessões de psicoterapia.

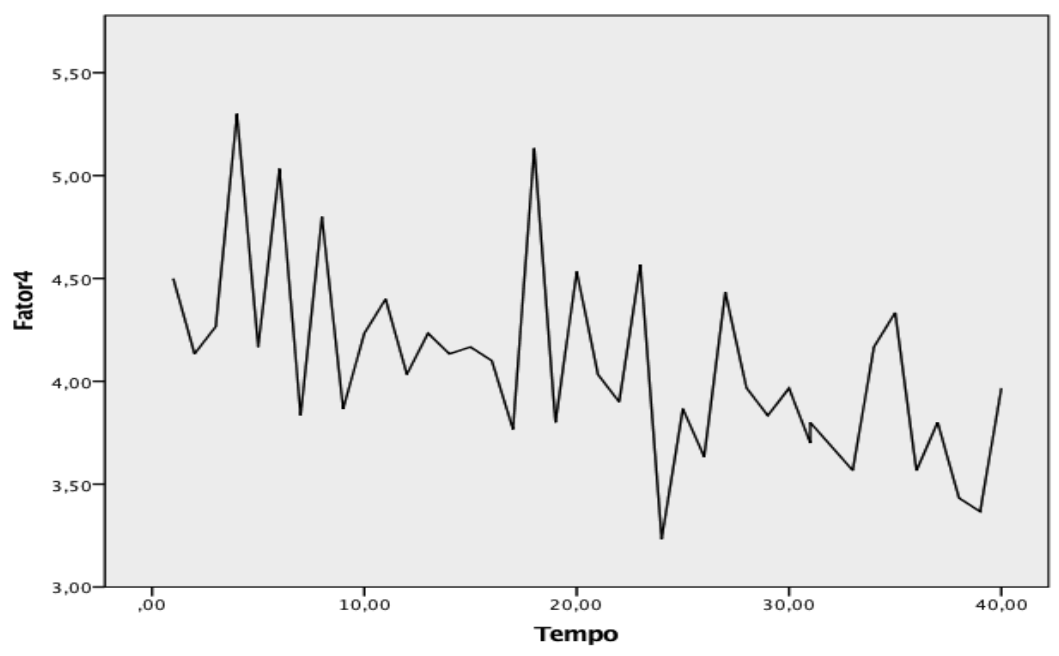

Figura 2. Variação da estrutura de interação 4 ao longo das 40 sessões de psicoterapia. 


\section{Discussão}

Observa-se que, no geral, o processo terapêutico de Alice caracterizou-se pela tentativa constante, por parte da terapeuta, de auxiliar a paciente a expressar suas emoções, encorajar suas verbalizações, procurando estabelecer conexões entre seus sentimentos e experiências. Isso era feito de maneira sensível e atenta ao nível de desenvolvimento da criança. É importante lembrar que, entre os sintomas que trouxeram Alice para tratamento, havia somatizações e que a menina atravessava um período de luto pela perda do seu pai. No trabalho com crianças, características como envolvimento afetivo, uma abordagem imparcial, sintonia linguística e afetiva podem ser necessárias em um grau maior do que se pode observar em modelos de tratamentos com adultos (Goodman, 2015; Palmer, Nascimento, \& Fonagy, 2013).

Tais características de intervenção são compatíveis com o modelo da PP (Zavaschi, Conte, Recondo, Bassols, \& Ghelen, 2008). Diante de um paciente com dificuldades de lidar com sentimentos dolorosos e/ou expressá-los, resultando em sintomas somáticos, uma das primeiras tarefas do terapeuta, além de estabelecer um bom vínculo e aliança terapêutica, será trabalhar no sentido auxiliar o paciente a expressar e verbalizar suas emoções e seus afetos.

Alice parece ter respondido bem a tais intervenções, pois, de acordo com o CPQ, sentia-se compreendida pela terapeuta, considerava seus comentários e observações e não se manteve distante dos seus sentimentos. Os itens mais e menos característicos do CPQ permitem levantar a hipótese de que foi estabelecido um vínculo positivo entre paciente e terapeuta, e uma boa aliança de trabalho.

Foram identificadas cinco EI na psicoterapia de Alice, de acordo com a análise fatorial de componentes principais. Algumas indicam uma matriz de transferência-contratransferência claramente positiva, como por exemplo a EI 1, enquanto outras uma matriz de transferência-contratransferência negativa, como por exemplo as EIs 3 e 4. Esses achados são semelhantes a outros estudos sobre EI na PP de crianças que também identificaram matrizes de transferência- -contratransferência positivas e negativas (Goodman, 2015; Goodman \& Athey-Lloyd, 2011; Ramires et al., 2015).

A EI 1 revela uma terapeuta sintonizada, com postura interpretativa, interagindo com uma criança ativa, expressiva. Separações e interrupções na psicoterapia foram discutidas e o brinquedo foi utilizado pela paciente de maneira fluída. É importante considerar dois fatores peculiares que marcaram a psicoterapia de Alice: a gravidez da terapeuta e seu afastamento durante a licença maternidade, e a morte do pai da menina, que antecedeu a busca pela psicoterapia. Levanta-se a hipótese que, no decorrer do tratamento, sempre que a EI 1 fez-se presente de maneira mais intensa, tais fatores puderam ser trabalhados, especialmente pela via da relação transferencial, como mostram os itens 69 e 96, presentes nesse padrão de interação. A importância do jogo também pode ser observada nessa EI (item 29), o que vai ao encontro da literatura de psicoterapia de crianças, que destaca o uso do brinquedo para se trabalhar as problemáticas da criança e a relação terapêutica (Zavaschi et al., 2008). Essa EI teve sua magnitude intensificada ao longo do tratamento, o que sinaliza para uma evolução positiva do mesmo e que é corroborado pela superação dos sintomas iniciais por parte de Alice.

A segunda EI descreve um padrão de relacionamento em que a menina se mostrava mais retraída e distante. A terapeuta assumiu uma postura mais ativa e apoiadora, e buscou se ajustar às necessidades da paciente. A terapeuta de crianças, por vezes, exerce na transferência um papel materno, de orientação, acolhimento e suporte, principalmente quando os adultos que as rodeiam são frágeis e não conseguem exercer esse papel (Luz, Keidann, \& Dal Zot, 2006; Zavaschi et al., 2008). Uma hipótese que pode ser levantada é que o fato da mãe da menina estar em luto pela perda do marido, associado à sua necessidade de sustentar emocionalmente e financeiramente sozinha a família, possa ter favorecido tais intervenções e também uma maior sensibilidade frente às dificuldades da criança, com menos neutralidade nessas situações.

As EIs 3 e 4 revelam interações pautadas por matrizes de transferência-contratransferência negativas. Alice se mostrava, nesses momentos do 
processo terapêutico, mais agressiva e defensiva (EI 3), ou mais resistente e dependente (EI 4). A terapeuta assumiu uma postura didática e diretiva nessas interações, e menos neutra. A expressão dos afetos negativos e dos impulsos agressivos faz parte do processo terapêutico psicodinâmico (Carlberg, 2009). No caso de Alice, que enfrentava o luto pela perda do pais, tais impulsos e afetos haviam sido fortemente reprimidos, o que resultou nos sintomas somáticos e dificuldades de adaptação na nova escola. Adicionalmente, a terapeuta engravidou e se afastou durante a psicoterapia, o que também pode ter contribuído para a resistência e defensividade da menina.

Alice mantinha uma relação de forte apego ao pai quando ele era vivo. Sua morte prematura e repentina pode ter mobilizado na menina fantasias e sentimentos de culpa. Consequentemente, ela se mostrava na escola bastante ansiosa e perfeccionista, com medo de errar. Na psicoterapia, conforme relatado pela terapeuta, manifestava desejo e preocupação de agradar e ser aceita, o que pode ter resultado no comportamento dependente e envergonhado (EI 4), defensivo (EI 3) e retraído (EI 2).

Em contrapartida, da parte da terapeuta, sua gravidez pode ter mobilizado sentimentos inconscientes de culpa, pelo fato de ter que "abandonar" sua pequena paciente que atravessava um importante luto. Isso pode ter favorecido sua menor neutralidade nessas EIs, e a adoção de posturas mais ativas e diretivas. A literatura sobre a gravidez das terapeutas na psicoterapia psicanalítica indica que, de alguma forma, esse fator impacta o processo terapêutico, podendo conduzir a mudanças nas suas atitudes e abordagem técnica (Schmidt, Fiorini, \& Ramires, 2015).

A EI 4 diminuiu de forma estatisticamente significativa no decorrer do tratamento. Levanta-se a hipótese de que foi possível a compreensão e elaboração, em alguma medida, desse padrão de interação no decorrer do processo. Jones (2000) afirmou que a mudança e os resultados na psicoterapia psicanalítica estão relacionados à compreensão e interpretação, por parte do terapeuta e do paciente, das suas EI, especialmente daquelas que revelam padrões conflitivos.

A EI 5 descreve um padrão de interação em que a paciente se mostrava tensa, ansiosa, e a te- rapeuta receptiva. Suas intervenções buscavam explorar e aprofundar conteúdos, sem focalizar defesas ou adotar estratégias mais interpretativas. Houve trabalho sobre separações (pausas, interrupções no tratamento) e sobre o relacionamento com pessoas significativas na vida da criança. Levanta-se a hipótese de que essa EI tenha constituído um momento preparatório para outros em que foi possível o uso de interpretações, cuja presença no tratamento de Alice também foi constatada. Sabe-se que a possibilidade de utilizar o recurso da interpretação é algo que necessita ser construído pelo paciente e pelo terapeuta na PP, requerendo, muitas vezes, um longo percurso até que esse entendimento será possível e constituirá uma consequência natural do processo empreendido até aquele ponto (Ferro, 1995).

A análise das EIs na psicoterapia de Alice e sua proximidade, em distintos momentos, a diferentes abordagens terapêuticas, pode ser compreendida à luz das contribuições de Luyten, Blatt e Mayes (2012). Esses autores compreendem o relacionamento terapêutico como um elemento central no tratamento, e o processo de mudança terapêutica como uma série de experiências de compatibilidade e incompatibilidade nesse relacionamento. Da mesma forma que uma mãe tentando compreender e responder às emoções e necessidades do seu infante, um terapeuta precisa ir ao encontro das demandas e dificuldades do seu paciente. Nessa direção, Palmer et al. (2013) constataram que muito do que eles identificaram "como aplicações não tradicionais de psicoterapia psicanalítica pode realmente acabar sendo os métodos mais usados de praticar estas ideias terapêuticas" (p. 175), e eles questionam "Por que não?"

Goodman (2015), em seu estudo sobre as EIs na PP de uma menina diagnosticada com transtorno de personalidade borderline, encontrou evidências preliminares da efetividade de estratégias de intervenções correspondentes ao modelo da terapia cognitivo-comportamental (TCC) para diminuir a raiva da paciente e suas explosões de emoções, substituídas mais tarde por estratégias de intervenção de PP. Podese observar, no presente processo terapêutico, assim como naquele descrito por Goodman (2015) e por Ramires et al. (2015), semelhanças 
em algumas EIs em diferentes psicoterapias de crianças com diferentes diagnósticos, nas quais uma criança difícil, agressiva, resistente ou distante se relaciona com uma terapeuta diretiva, didática ou menos neutra. Cada díade é única $\mathrm{e}$ não se espera que diferentes psicoterapias com crianças que apresentam diagnósticos distintos apresentem as mesmas EI. O que parece haver em comum nos tratamentos dessas crianças com Transtorno de Personalidade Borderline (Goodman, 2015), Transtorno de Asperger (Ramires et al., 2015) e TA do presente estudo é uma dificuldade importante de identificar e regular emoções. Assim, uma hipótese explicativa para a presença de intervenções correspondentes a diferentes abordagens é o fato de que ajudar uma criança a identificar, compreender e regular suas emoções demanda outras estratégias que não o trabalho interpretativo, pelo menos numa base preliminar.

Além disso, também é importante considerar a contribuição do terapeuta para as EIs. Goodman (2015) e Goodman e Athey-Lloyd (2011) mostraram que o terapeuta contribui para esses padrões de interação. Eles analisaram, em cada estudo, o tratamento de uma criança com dois diferentes terapeutas sequencialmente e constataram que cada terapeuta teve uma contribuição independente para a configuração das EIs, cuja magnitude diferiu entre ambos. Fatores como personalidade, conhecimento, experiência, sentimentos em relação à criança, e tratamento pessoal podem afetar o relacionamento terapêutico de diferentes maneiras com diferentes pacientes. No caso de Alice, é importante lembrar a variável da gravidez da terapeuta, que teve o seu primeiro bebê durante o processo terapêutico da menina. Tal fator também pode ter contribuído para a adoção de determinadas abordagens técnicas no decorrer do processo.

Após 40 sessões, que se estenderam por aproximadamente 15 meses, Alice não preenchia mais os critérios para o diagnóstico de TA. Sentia-se mais à vontade na escola e havia superado os sintomas somáticos que a trouxeram para tratamento. Do ponto de vista de um diagnóstico psicodinâmico, começava a apresentar traços obsessivos que, na visão da sua terapeuta, justificavam a continuidade da psicoterapia por mais um período. Contudo, por solicitação da sua mãe, justificada por fatores objetivos importantes, o tratamento foi encerrado naquele momento. Para a família, os objetivos com o tratamento haviam sido atingidos.

\section{Considerações Finais}

Este estudo contribui para o campo de pesquisas acerca do processo de psicoterapia de crianças. A análise desse processo, da perspectiva das EI estabelecidas entre paciente e terapeuta, revelou-se frutífera e promissora, informando sobre elementos que podem ser esperados em processos terapêuticos semelhantes, com crianças da mesma faixa etária e diagnóstico.

O método $\mathrm{Q}$ e o procedimento utilizado nesse estudo, o CPQ, mostraram-se adequados para o estudo do processo terapêutico. Novos estudos de caso sistemáticos, explorando a ação terapêutica e as EI em psicoterapias de crianças de diferentes faixas etárias e distintos diagnósticos poderá adicionar elementos para a formulação de uma base de evidências da PP de crianças.

$\mathrm{O}$ uso de medidas de resultado, alicerçadas em diferentes constructos, com foco nos sintomas, nas defesas, nos padrões de apego das crianças, por exemplo, também poderá contribuir para essa base de evidências, assim como a adoção de delineamentos quantitativos e estudos randomizados controlados. Uma limitação desse estudo foi não examinar os resultados da psicoterapia, com base na aplicação sistemática de medidas para esse fim.

A constatação da forma como o processo terapêutico se constrói a partir da díade é importante e pode auxiliar pesquisadores e psicoterapeutas a compreender as particularidades de cada psicoterapia. Também ficou clara a contribuição da terapeuta no processo. Apesar de todo psicoterapeuta adotar determinada orientação teórica, parece que, durante cada psicoterapia, ele precisar aderir ao paciente, sobretudo, mostrando-se sintonizado com as demandas de cada criança em particular. Isso pode implicar a adoção de abordagens técnicas que correspondem, pelo menos teoricamente, a abordagens terapêuticas que não a sua.

Uma tendência revelada pelo presente estudo e por outros disponíveis na literatura cientí- 
fica e aqui citados parece ser a adoção de uma abordagem integrativa na PP de crianças. O estudo empírico das EI contribui para a compreensão do que efetivamente ocorre no setting clínico desvelando, para além do que os terapeutas acreditam que praticam, o que efetivamente fazem no seu trabalho clínico com crianças.

\section{Referências}

Ablon, S. J., \& Jones, E. (2005). On analytic process. Journal of American Psychoanalytic Association, 53(2), 541-568.

American Psychiatric Association. (2013). Diagnostic and statistical manual of mental disorders. Fifth Edition. Washington, DC: Author.

Carlberg, G. (2009). Exploring change processes in psychodynamic child psychotherapy: The terapist's perspective. In N. Midgley, J. Anderson, E. Grainger, T. Vuckovic-Nesic, \& C. Urwin (Eds.), Child psychotherapy and research: New approaches, emerging findings (pp. 100-112). Nova York: Routledge.

Cheung, M. W. L., \& Chan, W. (2002). Reducing uniform response bias with ipsative measurement in multiple-group confirmatory factor analysis. Structural Equation Modeling: A Multidisciplinary Journal, 9(1), 55-77. doi: http:// doi.org/10.1207/S15328007SEM0901_4

Christiansen, N. D., Burns, G. N., \& Montgomery, G. E. (2005). Reconsidering Forced-Choice Item Formats for Applicant Personality Assessment. Human Performance, 18(3), 267-307.

Coombs, M. M., Coleman, D., \& Jones, E. E. (2002). Working with feelings: The importance of emotion in both cognitive-behavioral and interpersonal therapy in the NIMH treatment of depression collaborative research program. Psychotherapy: Theory/Research/Practice/Training, 39(3), 233-244.

Couto, M., Farate, C., Ramos, S., \& Fleming, M. (2011). A metodologia $Q$ nas ciências sociais e humanas: O resgate da subjectividade na investigação empírica. Psicologia, 25(2), 0721. Recuperado em http://www.scielo.mec. pt/scielo.php?script $=$ sci_arttext\&pid $=$ S0874$-20492011000200001 \& \operatorname{lng}=p t \& t \operatorname{lng}=p t$

Edwards, D. J. A. (2007). Collaborative versus adversarial stances in scientific discourse: Implications for the role of systematic case studies in the development of evidence-based practice in psychotherapy. Pragmatic Case Studies in Psychotherapy, 3(1), 6-34.
Eizirik, C. L., \& Hauck, S. (2007). Psicanálise e psicoterapia de orientação analítica. In A. V. Cordioli (Ed.), Psicoterapias: Abordagens atuais (3. ed., pp. 151-166). Porto Alegre, RS: Artmed.

Ferro, A. (1995). A técnica na psicanálise infantil. Rio de Janeiro, RJ: Imago.

Goodman, G. (2015). Interaction structures between a child and two therapists in the psychodynamic treatment of a child with borderline personality disorder. Journal of Child Psychotherapy. 41(2), 141-161.

Goodman, G., \& Athey-Lloyd, L. (2011). Interaction structures between a child and two therapists in the psychodynamic treatment of a child with Asperger's disorder. Journal of Child Psychotherapy, 37(3), 311-326.

Goodman, G., Edwards, K., \& Chung, H. (2014). Interaction structures formed in the psychodynamic therapy of five patients with borderline personality disorder in crisis. Psychology and Psychotherapy: Theory, Research and Practice, 87, 15-31.

Goodman, G., Midgley, N., \& Schneider, C. (2015). Expert clinicians'prototype of an ideal child treatment in psychodynamic and cognitive-behavioral therapy: Is mentalization seen as a common process fator? Psychotherapy Research. doi: http://dx.doi.org/10.1080/10503307.201 5.1049672

Jones, E. E. (1988). Manual for the Psychotherapy Process $Q$-set. Unpublished manuscript, University of California at Berkeley, Berkeley, CA, USA.

Jones, E. E. (2000). Therapeutic action. A guide to psychoanalytic therapy. London: Jason Aronson.

Jones, E. E., \& Pulos, S. M. (1993). Comparing the process in psychodynamic and cognitive-behavioral therapies. Journal of Consulting and Clinical Psychology, 61, 306-316.

Kramer, U., De Roten, Y., Michel, L., \& Despland, J. N. (2009, September-October). Early change in defence mechanisms and coping in short-term dynamic psychotherapy: Relations with symptoms and alliance. Clinical Psychology \& Psychotherapy, 16(5), 408-417. doi: http:// dx.doi.org/10.1002/cpp.616

Luyten, P., Blatt, S. J., \& Mayes, L. C. (2012). Process and outcome in psychoanalytic psychotherapy research: The need for a (relatively) new paradigm. In R. A. Levy, J. S. Ablon, \& H. Kächele (Eds.), Psychodynamic psychotherapy research: Evidence-based practice and prac- 
tice-based evidence (pp 345-359). New York: Humana Press.

Luz, A. B., Keidann, C. E., \& Dal Zot, J. S. (2006). Contratransferência e os Fatores da Vida Pessoal do Psicoterapeuta e/ou psicanalista. In J. Zaslavsky \& M. J. P. Santos, Contratransferência: Teoria e prática clínica. Porto Alegre, RS: Artmed.

Midgley, N. (2007). Researching the process of psychoanalytic child psychotherapy. In E. Kennedy \& N. Midgley, Process and outcome research in child, adolescent and parent-infant psychotherapy: A thematic review (pp. 8-53). London: North Central London Strategic Health Authority.

Midgley, N., \& Kennedy, E. (2011). Psychodynamic psychotherapy for children and adolescents: A critical review of the evidence base. Journal of Child Psychotherapy, 37(3), 232-260.

Miller, L. A., \& Lovler, R. L. (2015). Foundations of Psychological Testing: A Practical Approach $\left(5^{\text {th }}\right.$ Ed.). New York: Sage.

Palmer, R., Nascimento, L. N., \& Fonagy, P. (2013). The state of the evidence base for psychodynamic psychotherapy for children and adolescents. Child and Adolescent Psychiatric Clinics of North America, 22, 149-214. doi: dx.doi. org/10.1016/j.chc.2012.12.001

Ramires, V. R. R., Carvalho, C., Schmidt, F. M. D., Fiorini, G. P., \& Goodman, G. (2015). Interaction Structures in the Psychodynamic Therapy of a Boy Diagnosed with Asperger's Disorder: A Single-Case Study. Research in Psychotherapy: Psychopathology, Process and Outcome, 18(2).

Ramires, V. R. R., \& Schneider, C. (2016). Psicoterapia de Crianças: Desenvolvimento da Versão em Português do Child Psychotherapy Q-Set. Psicologia: Teoria e Pesquisa, 32(3). doi: http:// dx.doi.org/10.1590/0102-3772e323218

Sadock, B. J., \& Sadock, V. A. (2007). Compêndio de Psiquiatria: Ciências do comportamento e psiquiatria clínica (9. ed.). Porto Alegre, RS: Artmed.

Schmidt, F. M. D., Fiorini, G. P., \& Ramires, V. R. R. (2015). Psychoanalytic psychotherapy and the pregnant therapist: a literature review. Resear- ch in Psychotherapy: Psychopathology, Process and Outcome, 18(2).

Schneider, C. (2004). The development of the child psychotherapy Q-set (Doctoral dissertation) Retrieved from www.ipa.org.uk/ images/.../153598midgleychap.pdf

Schneider, C., \& Jones, E. E. (2004) Child Psychotherapy Q-Set Coding Manual. Unpublished manuscript, University of California at Berkeley, Berkeley, CA, USA.

Schneider, C., \& Jones, E. E. (2012). Appendix IB. Child Psychotherapy Q-Set. Coding Manual. In R. A. Levy, J. S. Ablon, \& H. Kächele (Eds.), Psychodynamic psychotherapy research: Evidence-based practice and practice-based evidence (pp. 611-626). New York: Human Press.

Schneider, C., Midgley, N., \& Duncan, A. (2010). A "motion portrait" of a psychodynamic treatment of an 11-year-old girl: Exploring interrelations of psychotherapy process and outcome using the Child Psychotherapy Q-Set. Journal of Infant, Child and Adolescent Psychotherapy, 9, 94-107.

Schneider, C., Pruetzel-Thomas, A., \& Midgley, N. (2009). Discovering new ways os seeing and speaking about psychotherapy process: The Child Psychotherapy Q-Set. In N. Midgley, J. Anderson, E. Grainger, T. Vuckovic-Nesic, \& C. Urwin (Eds.), Child psychotherapy and research: New approaches, emerging findings (pp. 72-84). New York: Routledge.

Williams, P. (2007). What is psychoanalysis? What is a psychoanalyst? In E. Person, A. Cooper, \& G. Gabbard (Eds.), The American psychiatric publishing textbook of psychoanalysis. American Psychiatric Pub.

Zavaschi, M. L., Conte, C., Recondo, R., Bassols, A. M., \& Ghelen, M. (2008). Psicoterapia de orientação analítica na infância. In A. V. Cordioli (Ed.), Psicoterapias: Abordagens atuais. São Paulo, SP: Artmed. 